\title{
Prevalence and Correlates of Substance Use Among Young Asian Pacific Islander Men Who Have Sex with Men
}

Don Operario • Kyung-Hee Choi • Priscilla Lee Chu •

Willi McFarland • Gina M. Secura • Stephanie Behel •

Duncan MacKellar • Linda Valleroy

(C) Society for Prevention Research 2006

The DOI in the PDF file of the following article which was published in Prevention Science, Volume 7, Number 1 (March 2006 ) is incorrect. It should be 10.1007/s11121-005-0018-x instead of 10.100/s11121-005-0018-x. 